\title{
Efficient Technique for Model Order Reduction Retaining Non-Minimum Phase Characteristics using Clustering Dominant Pole-Zero Algorithm
}

\author{
Anuj Abraham ${ }^{1)}$, N. Pappa ${ }^{2)}$ and Daniel Honc ${ }^{3)}$ \\ 1) Department of Applied Electronics \& Instrumentation, Rajagiri School of Engineering \& Technology, APJ Abdul \\ Kalam Technological University, Kerala, India, e-mail: anuj1986aei@ gmail.com \\ ${ }^{2)}$ Department of Instrumentation Engineering, Madras Institute of Technology Campus, Anna University, Chennai, \\ India, e-mail: npappa@ rediffmail.com \\ ${ }^{3)}$ Department of Process Control, Faculty of Electrical Engineering and Informatics, University of Pardubice, \\ Pardubice, Czech Republic, e-mail: daniel.honc@upce.cz
}

\begin{abstract}
Model Order Reduction (MOR) challenges a high dimensional problem and plays a key role in areas where dynamic simulation studies are necessary for modern simulation strategy. Many conventional reduction methods namely, reduced order models based on Least Square Method (LSM), Balanced Truncation, Hankel Norm reduction, Dominant Pole Algorithm (DPA) and CDPA method have been developed in the field of control theory. Among these, recently proposed Clustering Dominant Pole Algorithm (CDPA) is able to compute the full set of dominant poles and their cluster center efficiently. In this paper, a hybrid algorithm for model order reduction known as Clustering Dominant Pole-Zero Algorithm (CDPZA) is proposed to identify and preserve the dominant zeros of the processes exhibiting non-minimum phase behaviour. The CDPZA method combines the features of clustering method and DPA. Further, the cluster centers of the dominant zeros in the numerator polynomial are determined using factor division algorithm. The Benchmark HiMAT system of $6^{\text {th }}$ order is considered for testing and validation of the proposed algorithm. The simulation studies are carried out to show the efficacy of the proposed algorithm over conventional MOR algorithms.
\end{abstract}

Keywords - dominant pole, dominant zero, non-minimum phase, model order reduction, balanced truncation, Hankel norm, clustering.

\section{INTRODUCTION}

MOR application areas include numerical analysis in linear and nonlinear system algebra, structural mechanics, parametric and uncertain systems and applied to aerospace, petroleum industries where rigorous design is important to deal with as a small dimension problem [1]. Dynamic behaviour of process models is utilized to decide the order of any simulated model.

The main advantage of reduction methods is that, it is applied to online applications with reduction in complexity and time elapsed for simulation and retains accuracy. The model reduction technique chosen is appropriate if the reduced order model response is analogous to the higher order model response.

The Truncated Balanced Realization (TBR) is a model order reduction technique that has been originated from the control theory based on the concept of controllability and observability [2]. In this technique, balanced realization of a dynamical system is performed to obtain the state variables that have equal controllability and observability [3]. As some of the states are hard to control and observe, this step is essential in a dynamical system. Thus, the truncation of these kinds of states leads to a reduced order model. The major drawback in the TBR method is that it does not preserve or guarantee stability; it also falls in the linear projection framework and does not provide an optimal approximation [4].

The Hankel norm approximation method is used especially in the area of control and system theory where the balanced state space model for the system is computed initially. But, the balancing transformations for system models having uncontrollable and unobservable states are generally singular. This results in practical difficulty and poses a serious drawback to use the standard Hankel norm approximation theory.

The behaviour of a large scale dynamical system can often be described by a relatively small number of its dominant modes. By the the state space projection on the subspace spanned by the dominant modes, the model equivalent can be obtained. Model approximation has been successfully applied to transfer functions of large scale power systems and electrical circuits for application to stability analysis.

Generally, most of the higher order systems are approximated to FOPDT or SOPDT systems. Based on the system nonlinearity, interactions the dynamics of the system variations and desired performances are not met. A good approximated model based on corresponding dominant poles of the system transfer function representing eigenvectors and eigenvalues is formulated. An eigenvalue method is required that computes the most dominant poles and corresponding modes. The Dominant Pole Algorithm (DPA) works only for stable systems with the above concept, where roots of the denominator polynomial represent nearby poles towards the origin [5]. A hybrid algorithm that combines the clustering method and DPA method captures the essential dynamics of the system. The CDPA method efficiently computes the poles that are more dominant in the system and retain the full order characteristic behaviour. The denominator 
polynomial of the reduced order model with respect to full order model is determined by forming the clusters of the dominant poles and the coefficients of numerator polynomial with respect to full order model are obtained by using the factor division algorithm. Similarly, in the proposed CDPZA algorithm, inverse transfer function is formulated and the dominant zeros and dominant poles are determined based on DPA and CDPA algorithm respectively. The dominant zeros in numerator polynomial are retained and the remaining coefficients are obtained by factor division algorithm [6]. The simulation results show the effectiveness of CDPA and CDPZA algorithms with the conventional existing MOR techniques available in the literature.

\section{CONVENTIONAL MOR TECHNIQUES}

\section{A. Least Square Method (LSM)}

The LSM incorporates parameterized model based identification methods which relates an observable variable $\mathrm{y}(\mathrm{t})$ to a regressor vector $\phi(\mathrm{t})$. If a model has an unknown parameter vector $\theta$, then its value can be estimated by the LSM [7]. The model structure with a linear relationship is given by

$$
\mathrm{y}(\mathrm{t})=\phi . \theta,
$$

where $\phi(\mathrm{t})$ is also known as observation matrix.

Estimated or model output is given by $\hat{y}(t)=\phi^{T} \cdot \theta+\varepsilon(t)$. Hence, the prediction error residue $\varepsilon(\mathrm{t})=\hat{\mathrm{y}}(\mathrm{t})-\phi^{\mathrm{T}} \cdot \theta$, where $\phi^{\mathrm{T}} \cdot \phi$ is invertible.

Formulation of the objective function for error minimization is expressed, as in (1)

$$
\underset{\min }{\mathrm{J}(\theta)}=\sum_{t=1}^{N} \varepsilon^{2}(\mathrm{t})=\sum_{t=1}^{N}\left(\mathrm{y}(\mathrm{t})-\phi^{\mathrm{T}} \cdot \theta\right)^{2} .
$$

Hence, the estimated parameter vector is defined as in (2),

$$
\hat{\theta}=\left(\phi^{\mathrm{T}} \cdot \phi\right)^{-1} \phi^{\mathrm{T}} \mathrm{y}(\mathrm{t}) \text {. }
$$

As such, the LSM cannot be implemented for higher order systems as it is computationally intensive.

\section{B. Balanced Truncation}

The Balanced Truncation (BT) method guarantees an error bound on the infinity norm of the additive error $\left\|\mathrm{G}-\mathrm{G}_{\text {red }}\right\|_{\infty}$ for well-conditioned model reduced problems [8].

Given a state space $(\mathbf{A}, \mathbf{B}, \mathbf{C}, \mathbf{D})$ of a system and $k$, the desired reduced order, the following steps will produce a similarity transformation to truncate the original state space system to the $k^{\text {th }}$ order reduced model [9].

\section{BT Method}

Step 1: Find the Singular Value Decomposition (SVD) of the controllability and observability grammians.
Step 2: Calculate the square root of the grammians (left/right eigenvectors).

Step 3: Find the SVD to Step 2.

Step 4: Finally, the left and right transformation for the final $\mathrm{k}^{\text {th }}$ order reduced model is computed.

\section{Hankel Norm Approximation}

The Hankel norm of a system $\mathrm{G}=(\mathrm{A}, \mathrm{B}, \mathrm{C}, \mathrm{D}) \in \mathrm{H}_{\infty}$ is defined, as in (3),

$$
\|G\|_{\mathrm{H}}^{2}=\sup \frac{\int_{0}^{\infty} \mathrm{y}^{2}(\mathrm{t}) d t}{\int_{0}^{\infty} \mathrm{u}^{2}(\mathrm{t}) d t}
$$

where $\mathrm{y}(\mathrm{t})=\int_{-\infty}^{0} \mathrm{C} \mathrm{e}^{\mathrm{A}(\mathrm{t}-\mathrm{s})} \mathrm{Bu}(\mathrm{s})$.

The Hankel norm gives how much energy [10] can be transferred from past inputs into future outputs through the system $\mathrm{G}(\mathrm{s})$. In control theory, eigenvalues define system stability and Hankel Singular Values (HSV) defines the "energy" of each state in the system. Its characteristics in terms of stability, frequency, and time responses are preserved by keeping larger energy states of a system based on the HSV. This can achieve a reducedorder model that preserves the majority of the system characteristics. Mathematically, given a stable state-space system (A, B, C, D) its HSV are defined, as in (4)

$$
\|\mathrm{G}\|_{\mathrm{H}}^{2}=\sqrt{\lambda_{\max }}(\mathrm{PQ})=\sigma_{i}
$$

where $\sigma_{i}$ is the Hankel singular values.

The controllability and observability grammians $\mathbf{P}$ and $\mathbf{Q}$ respectively satisfy,

$$
\begin{aligned}
\mathbf{A P}+\mathbf{P} \mathbf{A}^{\mathrm{T}} & =-\mathbf{B} \mathbf{B}^{\mathrm{T}} \\
\mathbf{A}^{\mathrm{T}} \mathbf{Q}+\mathbf{Q A} & =-\mathbf{C}^{\mathrm{T}} \mathbf{C}
\end{aligned}
$$

One defines the Hankel operator $\Gamma_{\mathrm{G}}$ of the system $\mathrm{G}(\mathrm{s})$ by

$$
\begin{gathered}
\Gamma_{\mathrm{G}}: L_{2}(-\infty, 0]:\left(\Gamma_{G} \mathrm{u}\right)(\mathrm{t})= \\
\int_{-\infty}^{0} \mathbf{C} e^{\mathbf{A}(\mathrm{t}-\mathrm{s})} \mathbf{B} \mathrm{u}(\mathrm{s}) d s, \mathrm{t}>0
\end{gathered}
$$

This method also guarantees an error bound on the infinity norm of the additive error $\left\|\mathrm{G}-\mathrm{G}_{\text {red }}\right\|_{\infty}$ for wellconditioned model reduction problems as in the balanced truncation method [8] and [19].

$$
\left\|\mathrm{G}^{-\mathrm{G}_{\mathrm{red}}}\right\|_{\infty} \leq 2 \sum_{k+1}^{n} \sigma_{i}
$$

where $\sigma_{i}$ are singular values of a given system $\mathrm{G}(\mathrm{s})$. 


\section{Dominant Pole Algorithm (DPA)}

The DPA computes dominant poles of $\mathrm{G}(\mathrm{s})$ based on the Newton process [11]. A pole $\lambda_{i}$ that corresponds to a residue $R_{i}$ with large magnitude $\left|R_{j}\right|$ is called a dominant pole. A dominant pole is well observable and controllable in the transfer function. This can also be observed from the corresponding Bode Magnitude plot of $\mathrm{G}(\mathrm{s})$, where peaks occur at frequencies close to the imaginary parts of the dominant poles of $\mathrm{G}(\mathrm{s})$

$$
\mathrm{G}(\mathrm{s})=\mathbf{C}(\mathrm{s} \mathbf{I}-\mathbf{A})^{-1} \mathbf{B}=\sum_{i=1}^{n} \frac{R_{i}}{\mathrm{~s}-\lambda_{i}}
$$

where, residue $R_{i}=\left(\mathbf{C x}_{i}\right)\left(\mathrm{y}_{i} \mathbf{B}\right)$ and $\mathrm{x}_{i}, \mathrm{y}_{i}, \lambda_{i}$ are eigen triplets $(\mathrm{i}=1,2, \ldots \mathrm{n})$.

Consider a pole $\lambda=\alpha+\mathrm{j} \beta$, with residue $R$ then it is shown that,

$$
\begin{gathered}
\lim _{\omega \rightarrow \beta} \mathrm{G}(j w)=\lim _{\omega \rightarrow \beta} \frac{R}{[j w-(\alpha+j \beta)]}+\sum_{j=1}^{n-1} \frac{\mathrm{C}}{\left[j w-\lambda_{j}\right]} \\
\lim _{\omega \rightarrow \beta} \mathrm{G}(j w)=\frac{R}{\alpha}+\mathrm{G}_{\mathrm{n}-1}(j \beta)
\end{gathered}
$$

Hence, pole $\lambda_{j}$ is dominant if $\left|\frac{R_{j}}{\operatorname{Re}\left(\lambda_{j}\right)}\right|$ is large and causes peak in the Bode plot.

\section{PROPOSED CDPZA ALGORITHM}

The recently reported hybrid algorithm [12] namely, the Clustering Dominant Pole Algorithm (CDPA) and proposed hybrid algorithm known as Clustering Dominant Pole-Zero Algorithm (CDPZA), combines the features of the clustering method and dominant pole algorithm and effectively matches the full order system characteristics.

In both CDPA and CDPZA methods, the denominator polynomial of the reduced order model with respect to full order model is determined by forming the clusters of the dominant poles and the coefficients of the numerator polynomial are obtained by using the factor division algorithm [12].

The poles of the transfer function are the $\lambda \in \rightarrow \mathrm{C}$ for which $\lim _{s \rightarrow \lambda}|H(s)|=\infty$.

Consider now the function as expressed below,

$$
\begin{gathered}
G(s)=\frac{1}{H(s)}, \\
G^{\prime}(s)=-\frac{H^{\prime}(s)}{H^{2}(s)} .
\end{gathered}
$$

Solve $\mathrm{x}_{\mathrm{k}}$ from $\left(\mathrm{s}_{\mathrm{k}} \mathbf{E}-\mathbf{A}\right) \mathrm{x}_{\mathrm{k}}=\mathrm{b}$ and $\mathrm{y}_{\mathrm{k}}$ from $\left(\mathrm{s}_{\mathrm{k}} \mathbf{E}-\mathbf{A}\right) \mathrm{y}_{\mathrm{k}}=\mathrm{c}$. Then compute the new pole estimate as in (10),

$$
\mathrm{s}_{\mathrm{k}+1}=\mathrm{s}_{\mathrm{k}}-\frac{\mathrm{c} \mathrm{x}_{\mathrm{k}}}{\mathrm{y}_{\mathrm{k}} \mathbf{E} \mathrm{x}_{\mathrm{k}}}=\frac{\mathrm{y}_{\mathrm{k}} \mathbf{A} \mathrm{x}_{\mathrm{k}}}{\mathrm{y}_{\mathrm{k}} \mathbf{E} \mathrm{x}_{\mathrm{k}}} .
$$

In both CDPA and CDPZA methods, the dominant poles are grouped into several clusters and then replaced by the corresponding cluster-centers. By the Factor division algorithm, the coefficients of the numerator polynomial are determined [13]. Now, consider $\mathrm{n}^{\text {th }}$ order linear dynamic system described by the transfer function as in (11),

$$
G(s)=\frac{N(s)}{D(s)}=\frac{e_{0}+e_{1} s+e_{2} s^{2}+K+e_{n-1} s^{n-1}}{f_{0}+f_{1} s+f_{2} s^{2}+K+f_{n} s^{n}}
$$

where $\mathrm{e}_{\mathrm{i}}: 0 \leq \mathrm{i} \leq \mathrm{n}-1$ and $\mathrm{f}_{\mathrm{i}}: 0 \leq \mathrm{i} \leq \mathrm{n}$ are scalar constants.

The corresponding $\mathrm{k}^{\text {th }}(\mathrm{k}<\mathrm{n})$ order reduced model is synthesized as follows,

$$
\mathrm{G}_{\mathrm{k}}(\mathrm{s})=\frac{\mathrm{N}_{\mathrm{k}}(\mathrm{s})}{\mathrm{D}_{\mathrm{k}}(\mathrm{s})}=\frac{\mathrm{a}_{0}+\mathrm{a}_{1} \mathrm{~s}+\mathrm{a}_{2} \mathrm{~s}^{2}+\mathrm{K}+\mathrm{a}_{\mathrm{k}-1} \mathrm{~s}^{\mathrm{k}-1}}{\mathrm{~b}_{0}+\mathrm{b}_{1} \mathrm{~s}+\mathrm{b}_{2} \mathrm{~s}^{2}+\mathrm{K}+\mathrm{b}_{\mathrm{k}} \mathrm{s}^{\mathrm{k}}}
$$

where $\mathrm{a}_{\mathrm{i}}: 0 \leq \mathrm{i} \leq \mathrm{k}-1$ and $\mathrm{b}_{\mathrm{i}}: 0 \leq \mathrm{i} \leq \mathrm{k}$ are scalar constants.

Let $r$ real poles in one cluster be $\left(\mathrm{p}_{1}, \mathrm{p}_{2}, \mathrm{p}_{3} \ldots, \mathrm{p}_{\mathrm{r}}\right)$; then the Inverse Distance Measure (IDM) criterion identifies the cluster center, as in (15)

$$
\mathrm{p}_{\mathrm{c}}=\left\{\sum_{\mathrm{i}=1}^{\mathrm{r}} \frac{1}{\mathrm{p}_{\mathrm{i}}} / \mathrm{r}\right\}^{-1}
$$

where $\mathrm{p}_{\mathrm{c}}$ is the cluster center from $r$ real poles of the full order system.

The power series of the original $n^{\text {th }}$ order system can be expanded about $\mathrm{s}=0$ as shown below,

$$
\mathrm{G}(\mathrm{s})=\mathrm{C}_{0}+\mathrm{C}_{1} \mathrm{~s}+\mathrm{C}_{2} \mathrm{~s}^{2}+\mathrm{L}
$$

The power series expansion coefficients are determined as follows:

$$
\begin{gathered}
\mathrm{C}_{0}=\mathrm{e}_{0} / \mathrm{f}_{0}, \\
\mathrm{C}_{\mathrm{i}}=\frac{1}{\mathrm{f}_{0}}\left[\mathrm{e}_{\mathrm{i}}-\sum_{\mathrm{j}=1}^{\mathrm{i}} \mathrm{f}_{\mathrm{j}} \mathrm{C}_{\mathrm{i}-\mathrm{j}}\right], \mathrm{i}>0, \\
\mathrm{e}_{\mathrm{i}}=0, \mathrm{i}>\mathrm{n}-1 .
\end{gathered}
$$

The reduced $\mathrm{k}^{\text {th }}$ order model is written as:

$$
G_{k}(s)=\frac{N_{k}(s)}{D_{k}(s)}=\frac{\sum_{i=0}^{k-1} a_{i} s^{i}}{\sum_{i=0}^{k} b_{i} s^{i}} .
$$

In the proposed CDPZA algorithm, the dominant poles of $\mathrm{G}^{-1}(\mathrm{~s})$ are the dominant zeros of $\mathrm{G}(\mathrm{s})$. The inverse transfer function is formulated and the dominant zeros and 
dominant poles are determined based on DPA and CDPA algorithm respectively. The dominant zeros in the numerator polynomial are retained and the remaining coefficients are obtained by factor division algorithm.

CDPZA algorithm

Step 1 : Consider the inverse transfer function $\mathrm{G}^{-1}(\mathrm{~s})$

Step 2 : Repeat Steps 2 to 11 as in CDPA algorithm to determine the dominant poles of $\mathrm{G}(\mathrm{s})$

Step 3 : Repeat Steps 2 to 10 as in CDPA algorithm to determine the dominant poles of $\mathrm{G}^{-1}$ (s)

Step 4 : Dominant poles of $\mathrm{G}^{-1}$ (s) are the dominant zeros of $\mathrm{G}(\mathrm{s})$.

Step 5 : Dominant zeros determined in Step 3 are retained and cluster centres of the remaining zeros is calculated and replaced using factor division algorithm in the numerator polynomial.

\section{BENCHMARK HIMAT EXAMPLE}

The algorithms are tested on the HiMAT (Highly Maneuverable Aircraft Technology) benchmark example [14]. The state space realization of the HiMAT model has 6 states, with the first four states representing angle of attack $(\alpha)$ and attitude angle $(\theta)$ and their rates of change $(\mathrm{d} \alpha / \mathrm{dt}, \mathrm{d} \theta / \mathrm{dt})$ and the last two representing elevon and canard control actuator dynamics. Therefore, the model considered has one control input as elevon deflection $\delta$ e and one measured output as angle of attack alpha $(\alpha)$. The continuous transfer function $\mathrm{G}(\mathrm{s})$ for the model is chosen as

$$
\mathrm{G}(\mathrm{s})=\left[\frac{-5.124 s^{4}-1099 s^{3}-28390 s^{2}-568.5 s+24.08}{s^{6}+64.55 s^{5}+1167 s^{4}+3729 s^{3}+5495 s^{2}+1102 s+708.1}\right]
$$

The pole-zero spectrum of the $6^{\text {th }}$ order HiMAT system transfer function is shown in Fig.1. The plant poles are located at $\mathrm{p}_{1}=-30.4865+3.6785 \mathrm{i}, \mathrm{p}_{2}=-30.4865-$ $3.6785 \mathrm{i}, \mathrm{p}_{3}=-1.7308+1.4838 \mathrm{i}, \mathrm{p}_{4}=-1.7308-1.4838 \mathrm{i}$, $\mathrm{p}_{5}=-0.0596+0.3754 \mathrm{i}$ and $\mathrm{p}_{6}=-0.0596-0.3754 \mathrm{i}$. Similarly, plant zeros of the HiMAT example are located at $\mathrm{z}_{1}=-184.4448, \mathrm{z}_{2}=-30.0160, \mathrm{z}_{3}=-0.0409, \mathrm{z}_{4}=$ 0.0208 .

From Fig. 1, it is evident that the given $6^{\text {th }}$ order HiMAT system has all the poles lying on the left side of the s-plane and having conjugate poles. The poles $\mathrm{p}_{1}=$ $-30.4865+3.6785 \mathrm{i}$ and $\mathrm{p}_{2}=-30.4865-3.6785 \mathrm{i}$, are lying far away from the s-plane origin and takes fast response in decaying with less effect on the system characteristics. The constant coefficients of the denominator polynomial used in the various techniques have an important role to play in stability and performance of an LTI system, while the numerator coefficients also have an influence on the system response to applied inputs [15]. The plant zero $\mathrm{z}_{4}$ is lying on the right side of the splane, which exhibits non-minimum characteristics. Model based control schemes are effectively used in industrial application namely cement industry, coal mill industry etc., where accurate model coefficient estimation plays a crucial role in improving the closed loop system performances [16].

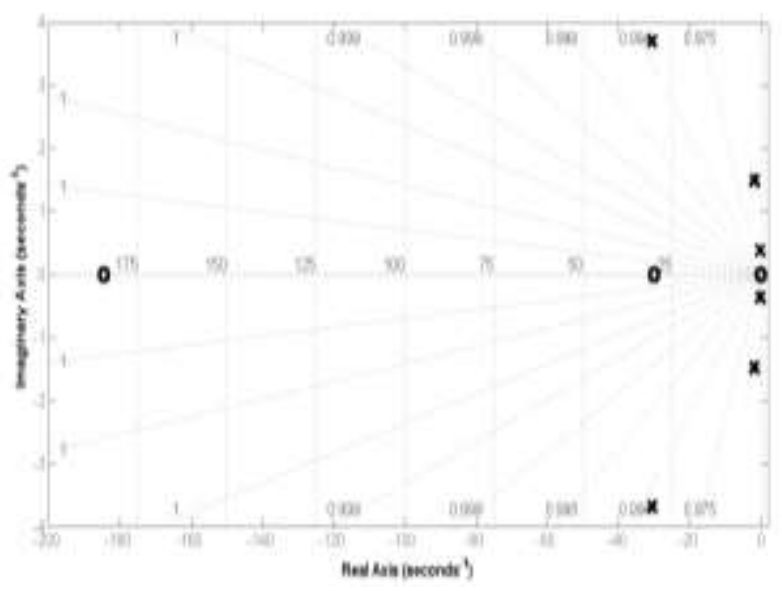

Fig. 1. Pole-Zero Spectrum of the $6^{\text {th }}$ order model of HiMAT example.

\section{RESULT AND DISCUSSIONS}

The optimal Hankel norm approximation gives the fourth order transfer function model. The transfer function displays a similar frequency response to the reduced order models. The gain characteristics of the reduced order models are compared in Fig. 3 with the $6^{\text {th }}$ order HiMAT system. The Hankel singular values of the $6^{\text {th }}$ order system are $\sigma_{1}=10.46, \sigma_{2}=8.22, \sigma_{3}=3.1, \sigma_{4}=1.1, \sigma_{5}=0, \sigma_{6}=0$, which means that fourth order model can be good approximants as reported in [12], [17]. The fourth order models using Hankel norm, DPA, CDPA and proposed CDPZA are determined. Whereas the third order model is obtained by truncating any 3 poles using the BT method. Similarly, the first order model and second order model are obtained by using the LSM method.

The step responses of different conventional reduced order model techniques and recently proposed CDPA and novel CDPZA method are compared with the $6^{\text {th }}$ order HiMAT system as it is shown in Fig. 2. It is observed that the CDPZA and CDPA method resembles the response of the $6^{\text {th }}$ order HiMAT system $\mathrm{G}(\mathrm{s})$.

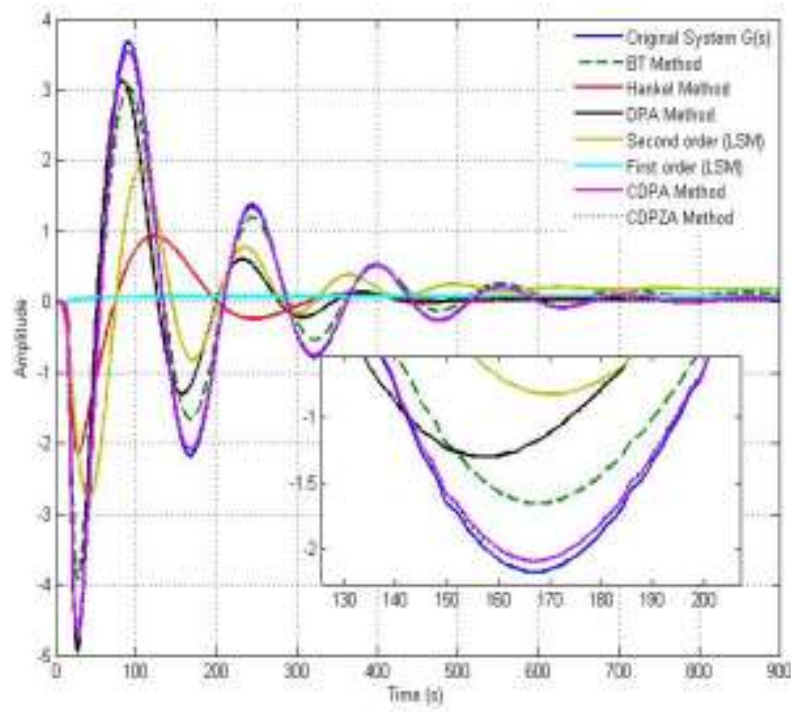

Fig. 2. Comparison of the step response of the $6^{\text {th }}$ order HiMAT system with various reduced order models. 

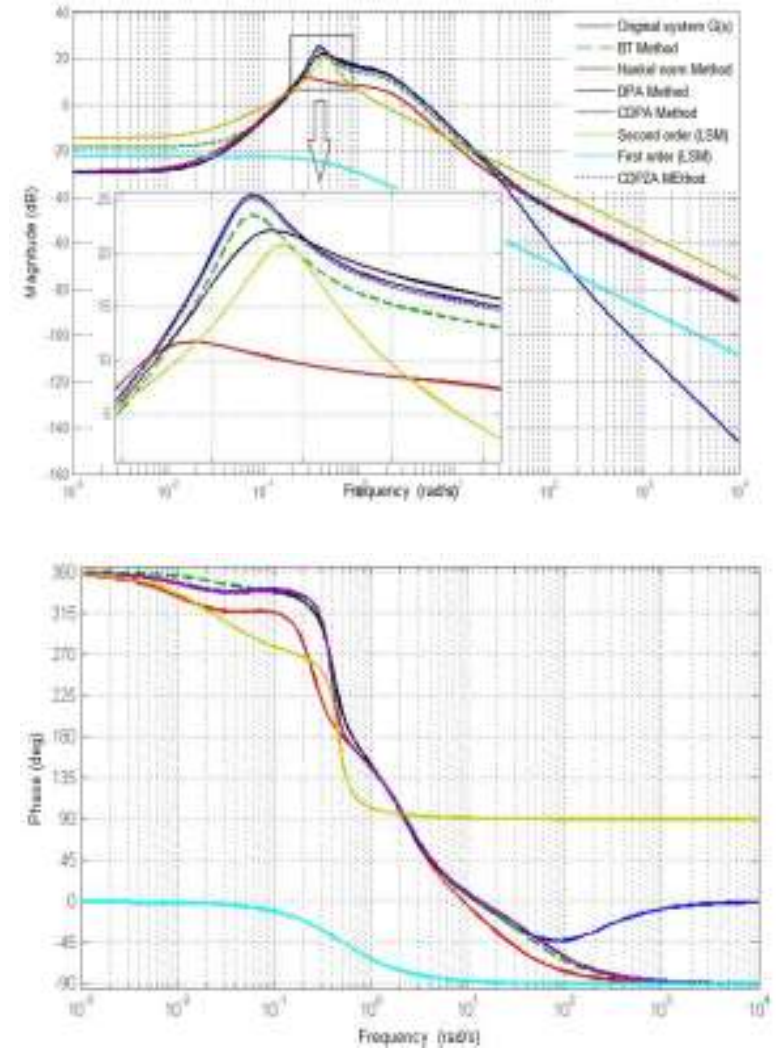

Fig. 3. Comparison of the gain and phase characteristics of the $6^{\text {th }}$ order HiMAT system with various reduced order models.

The gain G(s) and phase characteristics of the $6^{\text {th }}$ order HiMAT system with various reduced order models are compared in Fig. 3. It is observed that the essential dynamics of the system lie in the frequency range of $10^{-1}$ to $10^{1}$ radians/second (log scale) from the frequency response. The magnitude drops in both the very low and the high-frequency ranges. The result shows that the CDPA and CDPZA methods display similar characteristics with the $6^{\text {th }}$ order HiMAT system. Also, the step responses of hybrid algorithms are compared with the $6^{\text {th }}$ order HiMAT system separately as shown in Fig. 4. It is observed that the response of the proposed CDPZA method is better and resembles the $6^{\text {th }}$ order HiMAT system G(s) when compared with the CDPA method.

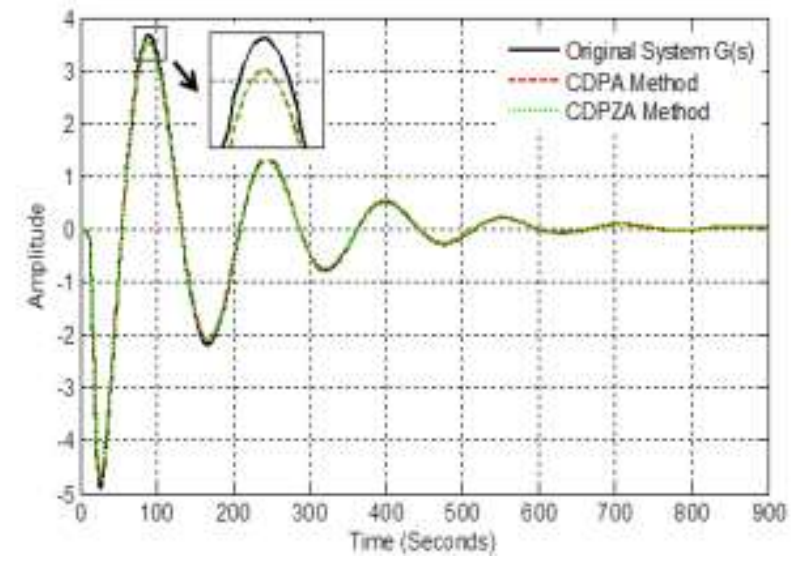

Fig. 4. Step responses of the $6^{\text {th }}$ order HiMAT system and reduced order hybrid models.

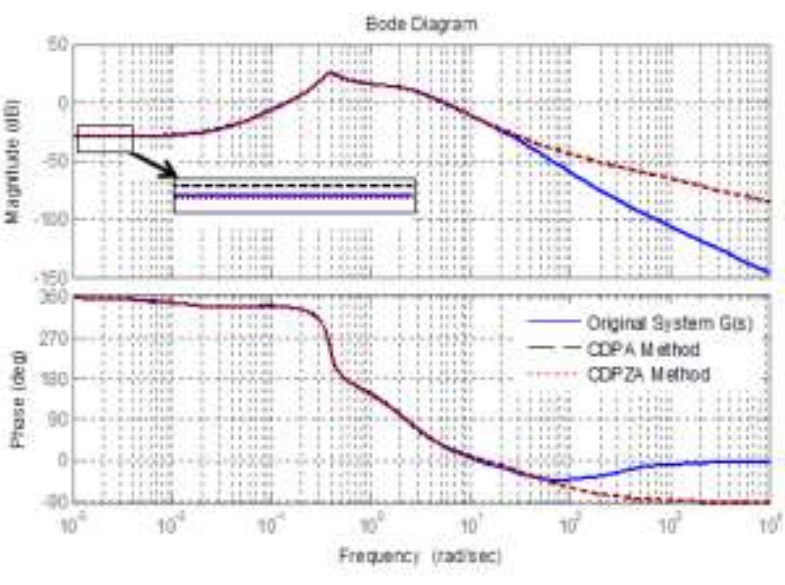

Fig. 5. Comparison of the gain G(s) and phase characteristics of the $6^{\text {th }}$ order HiMAT system and reduced order hybrid models.

The measure of comparison is made by computing the performance error index [18] known as Integral Square Error (ISE) between the $6^{\text {th }}$ order HiMAT system and reduced order models. The computed ISE values for various conventional and hybrid algorithms are listed in Table.1.

TABLE I.

COMPARISON OF ISE VALUES FOR VARIOUS MOR TECHNIQUES

Method $\quad$ Reduced models $\quad$ ISE

$$
\begin{array}{ll}
\begin{array}{l}
\text { First order } \\
\text { model using } \\
\text { LSM }
\end{array} & {\left[\frac{0.03817}{s+0.5168}\right]} \\
\begin{array}{l}
\text { Second order } \\
\text { model using } \\
\text { LSM }
\end{array} & {\left[\frac{-1.668 \mathrm{~s}+0.03828}{\mathrm{~s}^{2}+0.1525 \mathrm{~s}+0.2053}\right]} \\
\begin{array}{l}
\text { Third order } \\
\text { model using BT } \\
\text { method }
\end{array} & {\left[\frac{-8.024 \mathrm{~s}^{2}-4.57 \mathrm{~s}+0.1217}{\mathrm{~s}^{3}+1.752 \mathrm{~s}^{2}+0.7599 \mathrm{~s}+0.3462}\right]} \\
\begin{array}{l}
\text { Hankel norm } \\
\text { method }
\end{array} & {\left[\frac{0.62 \mathrm{~s}^{3}-19.80 \mathrm{~s}^{2}-0.56 \mathrm{~s}+0.04}{\mathrm{~s}^{4}+3.58 \mathrm{~s}^{3}+5.75 \mathrm{~s}^{2}+1.12 \mathrm{~s}+0.31}\right]} \\
\begin{array}{l}
\text { CDPA method } \\
\text { DPA }
\end{array} & {\left[\frac{0.56 \mathrm{~s}^{3}-29.07 \mathrm{~s}^{2}-0.64 \mathrm{~s}+0.03}{\mathrm{~s}^{4}+3.47 \mathrm{~s}^{3}+5.60 \mathrm{~s}^{2}+1.08 \mathrm{~s}+0.73}\right]} \\
& {\left[\frac{0.53 \mathrm{~s}^{3}-28.08 \mathrm{~s}^{2}-0.54 \mathrm{~s}+0.02}{\mathrm{~s}^{4}+3.34 \mathrm{~s}^{3}+5.21 \mathrm{~s}^{2}+1.42 \mathrm{~s}+0.71}\right]}
\end{array}
$$

\begin{tabular}{|c|c|}
\hline & $0.54 s^{3}-28.11 s^{2}-0.5657 s+0.024$ \\
\hline hod & $\overline{s^{4}+3.47 s^{3}+5.60 s^{2}+1.08 s+0.73}$ \\
\hline
\end{tabular}
0.080 


\section{CONCLUSIONS}

In this paper, a new hybrid algorithm mainly CDPZA method is proposed for Linear Time Invariant (LTI) systems. In this CDPZA method, the non-minimum phase behaviour is identified by retaining the dominant zeros and remaining roots in the numerator polynomial are determined by factor division algorithm. Also, in the recently proposed CDPA method, the denominator of the reduced model is synthesized by using clustering technique in which the dominant poles are grouped into several clusters and replaced by the corresponding cluster centers. The efficacy of the proposed algorithm is simulated with the help of the HiMAT benchmark example and it has been observed that it gives better responses with conventional MOR techniques in terms of error minimization. The simulated results show that the proposed method is simple, efficient to compute and retain dominant poles and dominant zeros that matches the $6^{\text {th }}$ order HiMAT benchmark system. Hence, the CDPZA algorithm is able to more closely follow the inverse characteristics exhibited by the non-minimum phase systems when compared with the other conventional reduction techniques.

\section{REFERENCES}

[1] S.K. Nagar and S.K. Singh, "An algorithmic approach for system decomposition and balanced realized model reduction," J. Franklin Inst., 2004, Vol. 341, pp. 615-630. https://doi.org/10.1016/j.jfranklin.2004.07.005

[2] M.G. Safonov and R.Y. Chiang, "A Schur Method for Balanced Model Reduction," IEEE Trans. on Automat. Contr., 1989, Vol. 34 (7), pp.729-733. https://doi.org/10.1109/9.29399

[3] David Amsallem, Matthew J. Zahr, and Charbel Farhat, "Nonlinear Model Order Reduction Based on Local Reduced-Order Bases," Int. J. Numer. Math. Eng., 2012, pp.1-30.

[4] Mihály Petreczky, et al., "Balanced truncation for linear switched systems," Elsevier, Nonlinear Analysis: Hybrid, 2013, Vol. 10, pp. 4-20. https://doi.org/10.1016/j.nahs.2013.03.007

[5] J. Rommes and N. Martins., "Efficient computation of transfer function dominant poles using subspace acceleration," IEEE Trans. Power System, 2006, Vol. 21 (3), pp. 1218-1226. https://doi.org/10.1109/TPWRS.2006.876671

[6] Vinod Kumar and J.P. Tiwari, "Order Reducing of Linear System using Clustering Method Factor Division Algorithm," International Journal of Applied Information Systems, 2012, Vol. 3, No. 5.
[7] S.J. Miller, The Method of Least Squares. Brown Univ., Providence, 2006.

[8] L.A. Aguirre, "Quantitative Measure of Modal Dominance for Continuous Systems," in Proc. of 32nd Conference on Decision and Control, 1993, pp. 2405-2410.

https://doi.org/10.1109/CDC.1993.325629

[9] Juergen Hahn and Thomas F. Edgar, "An improved method for nonlinear model reduction using balancing of empirical gramians," Computers and Chemical Engineering, 2002, Vol. 26, pp.13791397. https://doi.org/10.1016/S0098-1354(02)00120-5

[10]Huijun Gao, et al., "Hankel norm approximation of linear systems with time-varying delay: continuous and discrete cases," Int. J. Control, 2004, Vol. 77 (17), pp.1503-1520. https://doi.org/10.1080/00207170412331323641

[11]J. Rommes and N. Martins, "Computing transfer function dominant poles of large second-order dynamical systems," SIAM Journal on Scientific Computing, 2008, Vol. 30 (4), pp. 2137-2157. https://doi.org/10.1137/070684562

[12]Anuj Abraham, N. Pappa, Daniel Honc, and Rahul Sharma, "A hybrid method for determination of effective poles using clustering dominant pole algorithm," Int. J. of Math., Computational, Natural and Physical Eng., 2015, Vol. 7 (3), pp. 102-106.

[13]G. Parmar, S. Mukherjee, and R. Prasad, "System reduction using factor division algorithm and eigen spectrum analysis," Int. J. Applied Math. Modeling, 2007, Vol. 31, pp. 2542-2552. https://doi.org/10.1016/j.apm.2006.10.004

[14]M.G. Safonov, A.J. Laub, and G. Hartmann, "Feedback Properties of Multivariable Systems: The Role and Use of Return Difference Matrix," IEEE Trans. of Automat. Contr., 1981, AC- Vol. 26 (1), pp. 47-65.

[15]N. Martins, H.J.C.P. Pinto, and L.T.G Lima, "Efficient methods for finding transfer function zeros of power systems," IEEE Trans. Power Syst., 1992, Vol. 7 (3), pp. 1350-1361. https://doi.org/10.1109/59.207354

[16]A.C. Antoulas and D.C. Sorensen, "Approximation of large-scale dynamical systems: An overview," Int. J. Appl. Math. Comp. Sci., 2001, Vol. 11 (5), pp.1093-1121.

[17]N. Martins, L.T.G. Lima, and H.J.C.P. Pinto, "Computing dominant poles of power system transfer functions," IEEE Trans. Power Syst., 1996, Vol. 11 (1), pp. 162-170. https://doi.org/10.1109/59.486093

[18]A.K. Mittal, R. Prasad, and S.P. Sharma, "Reduction of linear dynamic systems using an error minimization technique," J. Inst. Eng. India IE(I) J. EL, 2004, Vol. 84, pp. 201-206.

[19]A. Abraham, N. Pappa, D. Honc, and R. Sharma, "Reduced order modelling and predictive control of multivariable nonlinear process," Sādhanā, 2018, Vol. 43 (3), pp. 1-18. https://doi.org/10.1007/s12046-018-0798-x 